\title{
Una aproximación al estado de la participación de los trabajadores en la innovación tecnológica en empresas de Cataluña
}

\section{Joaquín Juan Albalate}

Universitat de Barcelona. Departament de Sociologia i Anàlisi de les Organitzacions jjuana@ub.edu

\section{Resumen}

La participación de los trabajadores en la gestión de la tecnología es un fenómeno sociolaboral escasamente desarrollado. El análisis empírico de quince empresas del sector «auxiliar» del automóvil de Cataluña, mediante estudios de caso, ha permitido constatar que los niveles de participación de los trabajadores o de sus representantes en el proceso de innovación tecnológica son muy limitados, pues sólo en una de las empresas, el grado y la extensión de ese tipo de participación alcanzó su máxima expresión. Es decir, sólo en una de las quince empresas estudiadas, la toma de las decisiones relacionadas con las cuatro fases de que consta el proceso de innovación tecnológica (planificación, diseño, implantación y evaluación), se realizó con el grado de codecisión entre la parte social y la dirección, por lo que el control de los trabajadores o de sus representantes sindicales sobre ese proceso alcanzó el nivel más elevado de todos los previstos por esta investigación.

En las catorce empresas restantes, la distribución de los niveles de participación y, por tanto, de los tipos de control que se derivaron para los trabajadores fue la siguiente: en dos empresas, la participación en la gestión de la tecnología alcanzó un grado de tipo bidireccional (negociación con propuestas de ambas partes, aunque la dirección de la empresa se reservaba la última e irrevocable palabra), con una extensión de la participación hasta la fase de diseño de las tecnologías y, por tanto, incluyendo las fases de implantación y evaluación, con lo que los trabajadores accedieron a un control de tipo medio.

En otras seis empresas, el grado de participación alcanzado fue de tipo unidireccional (consulta optativa de la dirección a los trabajadores sin compromiso de aceptar sus propuestas), con una extensión que se limitaba a la fase de implantación de la tecnología — pues sólo en una de esas seis empresas los trabajadores pudieron participar en la fase de evaluación-, dando lugar a un tipo de control mínimo para los trabajadores. Finalmente, en las restantes seis empresas no se pudo detectar participación alguna, ni en grado ni en extensión.

Palabras clave: participación, trabajadores, tecnología, ideología.

\section{Abstract. An approach at situation of work participation in technology innovation of Catalonian companies}

The analysis of the worker's participation in the technological innovation process managing, through the study of cases in fifteen companies of the Catalonian industry of automotive components, made possible to state that the achieved participation levels are very 
limited. In only one of the fifteen companies studied, decisions taking related to the four steps composing the technological innovation process (planning, project, layout and evaluation) was performed with the adequate co decision levels between the social and management areas. On this way, in only one company, monitoring of workers or its representatives over such process, reached the highest expected level of this investigation.

Among the remaining companies, in two of them, the participation level in technology managing was of the bidirectional kind (negotiation between the two parts but final decision taken by management), while participation extend arrived just until project steps — consequently including layout and evaluation steps — resulting in a medium range control by the workers.

In other six companies, the achieved participation level was of the mono directional kind (management consults workers without any commitment of accepting proposals) with an extent limited to the layout step - except in one company where the extent reached the evaluation step- providing a minimum kind of technology control.

Finally, in the remaining six companies, no participation, either in level or extent was detected.

Key words: participation, workers, technology, ideology.

\section{Sumario}

Introducción

1. El trabajo de campo

2. Los resultados de la participación en la tecnología
3. Tipología organizacional de las empresas estudiadas

4. Algunas conclusiones

Bibliografía

\section{Introducción}

El contenido del presente artículo se corresponde con la síntesis de los principales resultados alcanzados con una investigación sobre la participación de los trabajadores de quince empresas «auxiliares» del automóvil, ubicadas en Cataluña, en el proceso de innovación tecnológica llevado a cabo en estas empresas. Estos resultados proceden del análisis efectuado, en una primera fase del trabajo de campo, en la tesis doctoral La participación de los trabajadores en la tecnología. Un enfoque sociocultural de la participación en empresas del sector "auxiliar» del automóvil de Cataluña, leída en la Universidad de Barcelona, en 2003.

Para obtener información necesaria, se recurrió a los estudios de caso, pues, a pesar de que esta técnica de investigación no permitía extrapolar los resultados alcanzados a toda la población a la que pertenecían los casos elegidos, se optó por ella porque era la que mejor se adaptaba al principal objetivo de la investigación: aprehender, con el máximo grado de profundidad, los razonamientos - a veces de difícil captura con técnicas cuantitativas - con los que los interlocutores habían de justificar sus discursos sobre la pertinencia o no de la citada participación.

Por otro lado, a todo ello se sumaba el hecho de que la hipótesis de llevar a cabo un procedimiento de análisis cuantitativo, utilizando una muestra aleatoria, quedaba, prácticamente, descartado, dado que la dimensión de las dos 
subpoblaciones de empresas en las que se iba a estudiar el fenómeno participativo eran lo suficientemente pequeñas (en total, 237 individuos estadísticos), como para que esa vía alternativa no resultara viable.

Sin embargo, y a pesar de la no aleatoriedad, se intentó que la selección de los quince casos de empresa respondiera a una serie de criterios de proporcionalidad, a fin de aproximarse en lo posible a un cierto grado de representatividad:

- En primer lugar, que el número de casos seleccionados en cada una de las poblaciones de empresas de los dos subsectores «auxiliares» elegidos (Producción de piezas y componentes metálicos y Producción e inyección de plástico y caucho), se acercara lo máximo posible al peso relativo de las poblaciones de ambos subsectores.

- En segundo lugar, que esa misma proporcionalidad se guardara, simultáneamente, con la distribución por segmentos de tamaño existente en las empresas de esas dos subpoblaciones, en términos de: menos de 50 trabajadores, entre 50 y 250 trabajadores y más de 250 trabajadores.

- En tercer y último lugar, que todos los casos elegidos lo fueran también de municipios con una elevada densidad de empresas de esos dos subsectores «auxiliares», sin que pudiera haber más de un caso de empresa de un mismo municipio.

\section{El trabajo de campo}

El acceso a la información que se deseaba para esa primera fase de la investigación se obtuvo de la aplicación de un guión semiabierto de entrevista pautada a los directivos de las citadas empresas, tras lo cual se pasó a su clasificación y a definir un indicador, atendiendo para ello a, entre otras fuentes, el esquema que utiliza el programa de investigación EPOC, de la Fundación Europea para la Mejora de las Condiciones de Vida y Trabajo (1996), de la Unión Europea, cuando analiza las políticas de participación en Europa. Este indicador quedó delimitado de la forma siguiente (cuadro 1).

Siendo:

1) Ausencia absoluta de información o existencia de información unilateral de la empresa sin derecho a réplica alguna.

2) Consulta de la empresa a los trabajadores, pero sin compromiso de ésta a aceptar las propuestas de aquéllos.

3) Consulta de la empresa negociada con los trabajadores, pero la decisión última es siempre de la empresa.

4) Decisión conjunta consensuada, con derecho a veto de ambas partes.

A la vez que:

a) Nula participación en fase alguna.

b) Propuestas de mejora tras la fase de implantación de la tecnología (en el presente) o/y en la fase de evaluación (de cara al futuro). 
Cuadro 1. Tipos de participación de los trabajadores en la tecnología.

Grado con que se participa

(intensidad o grado de la participación

en el proceso de innovación)
Cómo y dónde se participa

(extensión o formas de la participación en las diversas fases del proceso de innovación)
1) Ausencia o información unilateral (Aus/Inf)

2) Consulta unidireccional (Cons/Unid)

3) Consulta bidireccional (Cons/Bid)

4) Codecisión (Codeci) a) Ausencia de formas de participación en fase alguna (Part/Nula)

b) Propuestas de mejora a posteriori, en la fase de implantación o/y en la de evaluación (Part/Mínima)

c) Propuestas a priori en la fase de diseño y de mejora a posteriori en implantación o/y evaluación (Part/Media)

d) Propuestas a priori en las fases de decisión y diseño y de mejora a posteriori en las de implantación y evaluación (Part/Máxima)

Fuente: elaboración propia, a partir de EPOC (1996).

El proceso de innovación tecnológica que llevan a cabo las empresas se compone, fundamentalmente, de cuatro fases encadenadas: la de decisión o planificación, en la cual se llevan a cabo los estudios y las discusiones previos, antes de decidir la compra de la tecnología por parte de quienes posean el poder para tomar esa decisión, tras la cual sigue la etapa de diseño o selección, en la que se procede a elegir las características y prestaciones concretas que habrá de contener la tecnología. Tras ello, se sigue con dos etapas más, de carácter operativas y menos críticas que las dos anteriores: la de implantación, o puesta en marcha efectiva de la tecnología donde se ajusta y adapta al sistema sociotécnico de producción existente para pasar, finalmente, a la de evaluación, donde se lleva a cabo una valoración de los posibles problemas que puedan haberse detectado en el funcionamiento de la tecnología y, de ello, extraer las consecuencias necesarias de cara a la incorporación de nuevas tecnologías en el futuro (OIT, 1987: 10), la OCDE (OCDE, 1993: 105-125) y, particularmente, por el programa de investigación Participación directa en el cambio organizacional (EPOC, 1996), de la Fundación Europea para la Mejora de las Condiciones de Vida y Trabajo, de la Unión Europea. Ver también Fröhlich y otros (1990: 4).

c) Propuestas de mejora previas en la fase de diseño (anticipación parcial) y a posteriori, en implantación (presente) o/y evaluación (futuro).

d) Propuestas de mejora previas en las fases de decisión y diseño (anticipación íntegra), y a posteriori en las de implantación (presente) o/y evaluación (futuro).

\section{Los resultados de la participación en la tecnología}

Pues bien, la aplicación del anterior indicador a la información obtenida de las quince empresas estudiadas proporcionó los siguientes niveles de participación, en términos de grado y de extensión (cuadro 2). 
Cuadro 2. Distribución de las quince empresas, según el grado y las fases de la participación en la tecnología.

\begin{tabular}{|c|c|c|c|c|}
\hline \multicolumn{5}{|c|}{ Fases (extensión) } \\
\hline & $\begin{array}{l}\text { Ausencia } \\
\text { de participación } \\
\text { en fase alguna }\end{array}$ & $\begin{array}{l}\text { Participación } \\
\text { en las fases } \\
\text { de implantación/ } \\
\text { evaluación }\end{array}$ & $\begin{array}{l}\text { Participación } \\
\text { en las fases } \\
\text { de diseño } \\
\text { e implantación/ } \\
\text { evaluación }\end{array}$ & $\begin{array}{l}\text { Participación } \\
\text { en todas } \\
\text { las fases }\end{array}$ \\
\hline \multicolumn{5}{|l|}{ Grado (intensidad) } \\
\hline $\begin{array}{l}\text { Ausencia o inform. } \\
\text { unilateral }\end{array}$ & $\begin{array}{l}\text { Empresas 5, 6, 8, } \\
9,13,15\end{array}$ & $\begin{array}{l}\text { Empresas } 1,11 \text {, } \\
12\end{array}$ & & \\
\hline Consulta unidireccional & & Empresas 3, 4, 10 & & \\
\hline Consulta bidireccional & & & Empresas 2, 14 & \\
\hline Codecisión & & & & Empresa 7 \\
\hline
\end{tabular}

Fuente: elaboración propia.

La recapitulación de la información contenida en el cuadro 2 permitió comprobar que la situación de la participación en la tecnología en las quince empresas estudiadas se había distribuido de la siguiente forma:

- En seis de las quince empresas estudiadas (empresas 5, 6, 8, 9, 13 y 15) no se pudo detectar participación alguna, ni en grado ni en extensión.

- En tres de las nueve empresas restantes (empresas 1, 11 y 12) se registró una extensión de la participación mínima en la fase de implantación, pero con un grado de tipo nulo: "Ausencia de información/Información unilateral».

- En otras tres (empresas 3, 4 y 10), se registró la misma extensión de la participación — salvo en la empresa 3, en la que se amplió a la fase de evaluación-, aunque con un grado de "Consulta unidireccional» previa a los trabajadores.

- Por último, mientras que en otras dos empresas (empresas 2 y 14) se logró una participación con un grado de tipo "Consulta bidireccional», y una extensión que alcanzó a la fase de diseño de la tecnología, tan sólo en una empresa (empresa 7), se pudo detectar una participación con el grado de codecisión, aunque limitado al volumen de la inversión, con una extensión igualmente máxima, es decir, que abarcaba a todas las fases del proceso de innovación tecnológica.

\section{Tipología organizacional de las empresas estudiadas}

Tras ver la distribución de los niveles de participación alcanzados por los trabajadores en el proceso de innovación tecnológica en las quince empresas estudiadas, se pasó a hacer corresponder esos niveles con una determinada tipología organizacional, en función de cuál había sido su alcance en cada una de dichas empresas. 
Cuadro 3. Tipología organizacional de las empresas estudiadas, según la participación alcanzada por los trabajadores en la tecnología.

\begin{tabular}{lcccc}
\hline & $\begin{array}{c}\text { Empresas } \\
\text { «tayloristas» }\end{array}$ & $\begin{array}{c}\text { Empresas } \\
\text { «gerencialistas» }\end{array}$ & $\begin{array}{c}\text { Empresas } \\
\text { «humanistas- } \\
\text { paternalistas» }\end{array}$ & $\begin{array}{l}\text { Empresas } \\
\text { «democráticas» }\end{array}$ \\
\hline Empresas & $\begin{array}{c}\text { Empresas 5, 6, } \\
8,9,13,15\end{array}$ & $\begin{array}{l}\text { Empresas 1, 11, } \\
\text { Empresas 2, 14 }\end{array}$ & Empresa 7 \\
\hline $\begin{array}{l}\text { Control } \\
\text { de los trabajadores } \\
\text { sobre tecnología }\end{array}$ & Nulo & Mínimo & Medio & \\
\hline
\end{tabular}

Fuente: elaboración propia.

Para ello, se definieron cuatro «tipos ideales» de modelos de gestión empresarial («taylorista», "gerencialista», "humanista-paternalista» y «democrático») — debidamente justificados en la investigación - a partir del espectro de los principales rasgos identitarios que caracterizan y distinguen a esos modelos, en cuanto a su mayor o menor propensión hacia la participación, en particular la relacionada con la tecnología.

Finalmente, se pasó a comparar esos cuatro modelos de gestión con otros tantos tipos de control sobre la tecnología que resultaron para los trabajadores — «nulo», «mínimo», «medio» y «elevado»—, deducidos, a su vez, de los niveles de participación por éstos alcanzados.

Pues bien, de la información contenida en el cuadro 3, pudo deducirse que:

- Las empresas 5, 6, 8, 9, 13 y 15 respondieron con claridad a un modelo organizativo de carácter taylorista, asociado a un tipo de control para los trabajadores sobre la tecnología de índole nulo, puesto que no se pudo detectar participación alguna, ni en grado ni en extensión. Y eso es así, porque el modelo taylorista se caracteriza por concebir la tecnología como el mejor instrumento para incrementar la productividad laboral, mediante la automatización sucesiva de los procesos de producción, con el objetivo último de sustituir, progresivamente, el trabajo por capital.

Bajo esa lógica, la participación de los trabajadores, no sólo no tiene cabida porque la dirección se niega a admitir cualquier tipo de aportación o propuesta de los primeros, sino porque en el caso de ser aceptadas, lo que pueden conseguir los primeros es que se acelere aún más su sustitución, al incrementar la eficiencia de la tecnología con sus propuestas de mejora.

- Las empresas 1, 11 y 12 respondieron a un modelo organizativo de tipo gerencialista, por la «banda baja», pues, a pesar de que se pudieron detectar ciertas prácticas participativas en la fase de implantación de la tecnología, todas ellas lo fueron a iniciativa de la propia empresa, aunque con ausencia total de información e interacción entre las dos partes, coinci- 
diendo en esto último con el modelo taylorista. Se trataba, pues, de empresas «híbridas», localizadas en la frontera entre el taylorismo más puro y el gerencialismo, en las que sólo se promueve la participación de los trabajadores, para alcanzar con más rapidez y menos resistencias de éstos, los mismos objetivos que busca el taylorismo.

De otra parte, las empresas 3, 4 y 10 respondieron a un modelo de gestión, prototípicamente gerencialista, en la medida que el nexo que existía entre el grado —en este caso de "Consulta unidireccional»— y la extensión (fase de implantación), se correspondía, coherentemente, con una concepción de la participación diferenciada a la del modelo taylorista, al no considerarse objetivo prioritario la sustitución del trabajo por la tecnología, porque la implicación de los trabajadores en la gestión del trabajo es percibida aquí como algo que puede ofrecer mayores resultados y ventajas para la empresa — incluidos los de orden menos material — que los que se consiguen con el modelo taylorista.

Aun así, y a pesar de las diferencias entre ambas situaciones, se trataba de dos versiones de un mismo modelo - el gerencialista-, por lo que el control —en este caso mínimo- que se derivaba para los trabajadores de la tecnología era bastante parecido en las dos situaciones.

- Las empresas 2 y 14 respondieron, por su parte, a un modelo organizativo de tipo "humanista-paternalista», asociado a un tipo de control para los trabajadores sobre la tecnología de índole medio. A pesar de que estas empresas compartían con las gerencialistas la prioridad productivista, como fin último de la participación en la tecnología, la mayor participación alcanzada —al incluir, no sólo las fases de implantación y evaluación, sino también la fase crítica del diseño de la tecnología - confirmaba la existencia de un estilo directivo distinto.

Sin ser plenamente democrático, ese estilo se distinguía de los anteriores por apostar por un clima de trabajo y unas relaciones sociales propicias a una mayor proximidad y confianza, en tanto que premisa para crear en los trabajadores las condiciones de una implicación subjetiva superior y, con ello, conseguir con mayor consenso y legitimidad los objetivos de la empresa, distanciándose así del utilitarismo y de la inmediatez economicista con que el gerencialismo se plantea la participación.

Ahora bien, a pesar de todo ello, ni esas relaciones sociales suelen ser ajenas a un cierto paternalismo, ni los beneficios de la «proximidad»son enteramente simétricos, puesto que la dirección de este tipo de empresas siempre se reserva para sí espacios exclusivos de poder, sobre los que invariablemente tiene la última palabra, como a la hora de decidir el diseño definitivo de la tecnología y, por supuesto, la compra y los objetivos que se quieren conseguir con ésta.

Finalmente, la empresa 7 fue la única que se aproximó a una tipología de organización "democrática» $y$, a pesar de que no respondió íntegramente al caso óptimo definido en esta investigación (grado de codecisión y participación plena en todas las fases del proceso de innovación tecnológica) 
—en razón al coste que pudiera suponer la compra de la tecnología—, esa aproximación fue muy alta, por lo que el tipo de control que resultó para los trabajadores sobre la tecnología fue de índole elevado. Y eso fue posible, gracias a la existencia de una predisposición de la dirección para alcanzar acuerdos consensuados y sólidos con la parte social sobre la gestión de la organización del cambio tecnológico y de las consecuencias que pudieran derivarse para el propio trabajo.

En realidad, el modelo de gestión "democrático» se caracteriza, a diferencia de lo que sucede con los modelos anteriores, por concebir a la tecnología como un objetivo en sí mismo, y no sólo como un medio. Sin ser incompatible con la búsqueda de la máxima productividad de la tecnología, se intenta compatibilizarla con las necesidades personales y sociolaborales que tienen los trabajadores, siendo la tecnología un instrumento que ha de facilitar esas necesidades, procurando que el trabajo se convierta en una experiencia cualificante y satisfactoria para aquéllos.

En este tipo de empresas, las relaciones sociales de mutua confianza, no sólo no quedan al criterio más o menos arbitrario de la voluntad de los directivos, como en el modelo precedente, sino que están formalizadas en normas equitativas para que la toma de las decisiones importantes se realice, conjuntamente, por consenso entre las dos partes.

En un contexto como éste, la participación en la gestión del proceso de innovación tecnológica, no sólo propicia el alcance del máximo grado y extensión, sino que puede dar paso a la codeterminación de otro tipo de decisiones de nivel superior a la tecnología, como las inversiones en otras facetas de las empresas, o la misma definición de los objetivos de éstas, si así lo acuerdan las dos partes, sin que ninguna de ellas pueda imponer sus criterios sobre la otra.

\section{Algunas conclusiones}

A la vista de los resultados obtenidos en la participación en el proceso de innovación tecnológica y en las respectivas tipologías organizativas de las quince empresas estudiadas del sector «auxiliar» del automóvil de Cataluña, se apuntaron una serie de comentarios y reflexiones, siempre teniendo presente que su validez sólo podía serlo para esas empresas, a pesar de que, con la debida precaución metodológica a que obliga el uso de técnicas cualitativas de investigación, pueden ser extrapoladas a otras empresas del citado sector "auxiliar» y de otros sectores de actividad, que compartan características similares con las empresas aquí estudiadas.

- En primer lugar, los resultados obtenidos en las empresas estudiadas por esta investigación informaban de la presencia de un elevado nivel de exclusión estructurada de los trabajadores de la participación en la innovación tecnológica. Y eso era así porque, cuando existía participación, lo hacía con un grado o una intensidad escaso, y sólo cuando las tecno- 
logías estaban ya funcionando. En otras palabras, en el 80\% de las empresas estudiadas, o no había participación o, si existía, se limitaba a las fases más operativas y menos comprometidas para los directivos de esas empresas.

La persistencia de una política de exclusión de la participación, incluso cuando la dirección aporta información, pero es insuficiente, incontestable o/y tardía (Larrea, 1994: 134), además de que puede ser interpretado por los trabajadores y sus representantes como un motivo para ocultarles decisiones que pueden ir en contra de sus intereses (Aragón, 1998: 125), podría dar lugar también, como contrapartida no deseada por algunos directivos, a que, por la misma razón, los propios trabajadores o sus representantes se desvinculen de cualquier corresponsabilidad para con los cambios y problemas que se suelen derivar, cuando se introduce la tecnología sin su concurrencia.

No obstante, este tipo de resultados no se aparta demasiado de las grandes tendencias detectadas en los países europeos a finales de los años ochenta (Cressey, 1990: 106). Estas tendencias ya indicaban que, salvo en los países escandinavos y algunos casos excepcionales de toma de decisiones conjunta en el resto de países, en la mayor parte de las empresas europeas se percibía a la tecnología como un instrumento de potestad exclusiva de la dirección, siendo ésta la principal responsable de que la participación estuviera concentrada en la fase de implantación y, por tanto, excluida del resto de fases del proceso de innovación tecnológica.

Si éste era el panorama general que existía en Europa a finales de la década de los ochenta y ha coincidido, en términos generales, con los resultados aquí obtenidos en la mayoría de las empresas estudiadas, tras el transcurso de más de una década, podría apuntarse como hipótesis — con las necesarias precauciones metodológicas y la posible influencia de otras variables, aquí no contempladas - que ese diferencial de años podría aproximarse al «desfase» temporal del retraso en el que se encuentra este tipo de participación en Cataluña, respecto de la media europea.

- En segundo lugar, los resultados obtenidos sugerían igualmente que, cuanto mayor era el grado de que disponían los trabajadores para participar en la tecnología, mayor era también la amplitud de la participación en el proceso de innovación tecnológica. En otras palabras, y como ejemplo, sólo era posible participar en todas las fases de ese proceso si, previamente, los trabajadores tenían reconocido el poder necesario para codecidirlo con la dirección. Lo contrario - poder participar en la tecnología sin tener reconocido ese poder - sólo fue posible detectarlo cuando esa participación era de poca entidad, normalmente iniciada a instancias de las propias empresas.

Por tanto, para que los trabajadores o sus representantes sindicales puedan participar en las fases que exigen un tipo de decisiones, técnicamente más complejas y, a su vez, más comprometedoras para los directivos (Lahera, 2001: 112), además de disponer de las habilidades técnicas y 
sociales necesarias para contraponer sus propuestas a las ofrecidas por la dirección, han de poseer también las atribuciones de poder pertinentes para que la participación en esas fases más "críticas» se pueda materializar efectivamente.

La supuesta incapacidad técnica de los trabajadores - a menudo esgrimida por algunos directivos - para poder participar en esas fases, responde más a razonamientos ideológicos y a intereses ligados a la perpetuación de las prerrogativas de poder (Levinson, 1977: 91), que a una verdadera incapacidad de aquéllos para tomar ese tipo de decisiones, pues hoy hay los suficientes recursos formativos y de otra índole como para cubrir la supuesta carencia de cualificación.

No obstante, la posesión de tales atribuciones para poder ejercer la participación se mostró sólo como una condición necesaria, pero no suficiente. A pesar de que en algunas empresas los trabajadores disponían de un cierto grado para participar - por ejemplo, el de «Consulta unidireccional» en la empresa 10 - mayor que el que disponían los trabajadores de las empresas tayloristas, no por ello la extensión de dicha participación alcanzó unos niveles superiores, pues, como se pudo comprobar, además de disponer de un mínimo grado de poder, los trabajadores y sus representantes también habían de «querer» aprovechar las oportunidades ofrecidas por ese poder. Y esto último se demostró tan importante como el "poder», pues, para «querer» participar, se requería la identificación con unas ciertas ideas y valores - analizados en una segunda fase de la investigación- responsables, en buen medida, de que se activara o no la voluntad de "querer» participar de algunos trabajadores.

- En tercer lugar, la ausencia de participación de los trabajadores de las empresas gerencialistas - en las empresas tayloristas era obvia - en la fase de evaluación (salvo en la empresa 3), vino a confirmar que, aun siendo una fase objeto de posible participación menos crítica para los directivos, que la que tiene lugar en las fases más estratégicas, tal participación seguía quedando, mayoritariamente, descartada como objetivo por los trabajadores, pero también por los directivos. Algo ya constatado en otros países de nuestro entorno, a principios de los noventa (Smits, 1993: 21).

Con este tipo de resultados, es fácil deducir que la importancia que los directivos conceden al diagnóstico evaluativo de los problemas que se generan de la explotación de las tecnologías, conjuntamente con los trabajadores o con sus representantes, sigue siendo muy escasa. De esa forma, se pierde la oportunidad para aprovechar el bagaje de aprendizajes y experiencias que éstos últimos acumulan durante el uso y la conducción de las tecnologías - en tanto que cualificación susceptible de beneficiar a ambas partespara acordar las nuevas prestaciones y características que habrán de tener las futuras tecnologías, una vez evaluados los problemas y las ventajas de las anteriores.

- En cuarto lugar, es posible intuir que los resultados detectados en esta investigación puedan haber sido el fruto de un cierto avance experimen- 
tado a lo largo de los últimos años ${ }^{1}$ Y es que algunas empresas aquí catalogadas como gerencialistas, podrían haber evolucionado durante los últimos años desde un taylorismo "puro" inicial, hacia otras formas de gestión más participativas como éstas últimas, bien, por propia voluntad planificada, bien por adaptación pragmática a los cambios impuestos por el entorno (Regini, 1992: 62), o incluso, hacia otras formas de participación todavía más comprometidas que las de tipo gerencialista, por lo que no es posible concluir con rotundidad que la participación tecnológica no haya sufrido avance alguno, desde que se inició el proceso de innovación tecnológica a mediados de la década de los ochenta, a pesar de los escasos resultados aquí detectados.

Sin embargo, aún admitiendo que se hubiese podido producir tal avance, lo que sí parece haber sucedido es que no ha existido una institucionalización definitiva de las prácticas participativas, dado el carácter informal, inestable y específico con que se han establecido esos posibles avances en cada empresa. En efecto, la precariedad de bastantes de los logros que se han podido conseguir en la participación en el cambio tecnológico de algunas de las empresas aquí estudiadas, podría conllevar a que algunos directivos se desdijeran y revocaran sus compromisos sin demasiados problemas.

La aparición de cualquier coyuntura de orden político, económico-financiero o institucional que pudiera poner en peligro el logro de unos determinados objetivos económicos, podría convertirse en un desafío a la participación tecnológica alcanzada en algunas empresas porque, igual que se implantó en su momento como instrumento para afianzar una mayor productividad, se podría suprimir en cualquier otro, si los beneficios de productividad que aquélla proporcionaba, dejaran de compensar los costes que generaba.

Éste podría ser, por ejemplo, el caso de algunas de las empresas catalogadas en esta investigación de gerencialistas (empresas 3, 4, 10, 11 y 12), puesto que la participación tecnológica que se desarrollaba, era meramente instrumental y de carácter informal, sin que existiera acuerdo alguno con los representantes sindicales para formalizarla de forma duradera, al menos allí donde esa representación existía (sólo en las empresas 10 y 11).

- En quinto y último lugar, insistiendo en la ausencia de datos empíricos comparables con este tipo de participación, el único estudio con el que se

1. Se trata de una intuición, porque es algo prácticamente imposible de saber, si se tiene en cuenta que no se había publicado — al menos hasta el momento en que se finalizó esta investigación — ningún estudio empírico sobre este tema específico con el cual poder establecer algún tipo de comparación, y menos aún, con una metodología parecida o equiparable a la que aquí se siguió, y que se haya desarrollado en el territorio español y, mucho menos aún, en el de Cataluña, que no fuera el que realizó la Fundación Europea para la Calidad de Vida y del Trabajo para diversos países europeos —incluida España- a finales de la década de los ochenta, a pesar de haberse limitado a detectar la participación en el grado de codecisión que existía en la fase de decisión del proceso de innovación tecnológica de las empresas españolas que se estudiaron. 
Cuadro 4. Comparación de los resultados de la participación en la fase de planificación/decisión por tanto por ciento de empresas.

\begin{tabular}{llll}
\hline & \multicolumn{3}{c}{ Estudios } \\
\cline { 2 - 4 } Grado part. & $\begin{array}{c}\text { España (estudio } \\
\text { de la Fundación, 1987) }\end{array}$ & $\begin{array}{l}\text { UE-12 (estudio } \\
\text { de la Fundación, 1987) }\end{array}$ & $\begin{array}{c}\text { Cataluña } \\
\text { (trabajo de campo, 2001) }\end{array}$ \\
\hline Codecisión & $5 \%$ & $10 \%$ & $6 \%$ \\
\hline
\end{tabular}

Fuente: elaboración propia a partir de datos de la FEMCVT.

pudo establecer una mínima comparación de los resultados obtenidos fue - como ya se ha apuntado en la anterior nota a pie de página número 1con el realizado por la citada FEMCVT (Fundación Europea para la Mejora de las Condiciones de Vida y de Trabajo) para los doce países que formaban la UE a finales de los ochenta, incluida España.

Aunque las técnicas de investigación y los criterios utilizados para clasificar la información por ese estudio no coincidían exactamente con los utilizados por esta investigación (Fröhlich y otros, 1990: 5-7), el aspecto específico de la participación en la tecnología que exploró dicha fundación (grado de «codecisión»), permitió ofrecer el siguiente cuadro comparativo (cuadro 4).

Tal como puede observarse, el cuadro 4 muestra los resultados de la participación tecnológica alcanzados por las dos investigaciones en el momento de realizar los respectivos trabajos de campo.

Así, la comparación entre el porcentaje que alcanzaba la participación en la tecnología en España (5\%) y la media de la UE (10\%) en el estudio de la FEMCVT, ponía de manifiesto el menor desarrollo que había en España de ese grado de participación en la fase de decisión (concretamente, un 50\% menor).

De otro lado, la comparación con los resultados obtenidos por esta investigación — para ese mismo grado de participación — con los de la citada fundación para España y para la UE, permitían comprobar que, transcurridos catorce años, tan sólo en un 6\% de las empresas estudiadas en Cataluña (es decir, en una sobre un total de quince), los trabajadores habían alcanzado los máximos niveles de participación posibles en empresas capitalistas que operan en el mercado. Por ello, a pesar del hipotético avance que pudiera haberse experimentado en la participación tecnológica durante los últimos años, no parece - al menos en aquélla que conduce a la necesidad de un mayor compromiso de las dos partes - que haya progresado suficientemente, como para acercarse a los niveles con los que se desarrolla este tipo de participación en el resto de Europa. 


\section{Bibliografía}

Aragón SÁnCHEZ, Antonio (1998). La participación de los empleados en la empresa. Madrid: Consejo Económico y Social.

Cressey, Peter (1990). "Nuevas tecnologías y participación de los trabajadores». Sociología del Trabajo, 9. Madrid.

FRÖHLICH, D. y otros (1990-1991). "Caminos hacia la participación en la Comunidad Europea». Sociología del Trabajo, 11, invierno. Madrid, p. 3-24.

FundaCiÓn EuROPEA PARA LA MEJORA DE LAS CONDICIONES DE VIDA Y DE TRABAJO, EPOC (1996). Acortar distancias: la participación directa en el cambio organizativo. Luxemburgo.

LAHERA, Arturo (2001). «La participación de los trabajadores en la empresa: ¿hacia la democratización de las relaciones industriales? Una propuesta metodológica de análisis». En Fernández STEINKO, Armando; LACAlle, Daniel. Sobre la democracia económica. La democracia en la empresa. Barcelona: Viejo Topo, Fundación de Iniciativas Marxistas.

LARREA GAYARRE, José (1994). «Innovación técnica o nuevas tecnologías». Cuadernos de Sección: Sociedad, Técnica y Tecnología, 1. Universidad del País Vasco.

LEvinson, Charles (1977). La democracia industrial. Madrid: Asociación para el Progreso de la Dirección.

OCDE (1993). Gestión de recursos humanos y tecnologías de fabricación avanzada. Madrid: Ministerio de Trabajo y Seguridad Social.

OIT (1987). La participación en la empresa, 1981-1985. Madrid: Ministerio de Trabajo y Seguridad Social.

REGINI, Marino (1992). «Los empresarios frente al problema del consenso». Sociología del Trabajo, 16, otoño. Madrid.

SMITS, Ruud E.H.M. (1993). «Situación actual de la evaluación de tecnología en Europa». Quaderns de Tecnologia, 7. Barcelona: Institut Català de Tecnologia. 